the sum of all $C$ 's containing $\alpha_{1}$ as argument, and so on; $\sum\left(\lambda_{1}\right)$ is the sum of all $C$ 's not containing $\alpha_{1}$, and so on. Then $C()=\sum\left(\lambda_{1} \lambda_{2} \cdots \lambda_{n+1}\right), C\left(\alpha_{1}\right)=\sum\left(\alpha_{1} \lambda_{2} \cdots \lambda_{n+1}\right)$ and so on. The $C$ 's are replaced by their corresponding expressions in terms of $\sum$ and the subsequent development follows that of an earlier paper reported in this Bulletin (abstract 47-9-430). The preceding provides a representation $R_{n}$ for the latter exposition; $R_{2}$ is equivalent to the so-called Euler diagram which in turn is topologically equivalent to the figure of a triangle in a descriptive plane extended in accordance with the author's system ${ }^{2} K_{2}$ (ibid., p. 395 , Axiom 12). $R_{2}$ is topologically equivalent to the figure of a trifoliate curve inscribed in a circle. Another basis for the algebra of logic in the author's theory is found in a simple modification of his axioms for a linear "permutation" (ibid., p. 370). (Received November 24, 1941.)

\title{
Statistics And Probability
}

88. R. D. Gordon: An application of the Cauchy integral to a problem in probability theory.

The author was consulted about interpretations of certain data on biological populations. The problem aims at obtaining error corrections on the data. The classical basis of the Bayes-Laplace formula is chosen: $(x)(n / x)=(n)(x / n)$ where the notation has the usual meaning in probability theory. The probability $(n / x)$ is known through its generating function. The distribution $(x)$ of the parameter $x$, an integer, is taken on reasonable grounds to be $(x)=1 / b+1$, for $0 \leqq x \leqq b ;(x)=0$ otherwise. Then $(n)=\sum(x)(n / x)$ is determined. An "observation" results in a value $n_{0}$ of the stochastic variable $n$. The problem: to determine the distribution $\left(n / n_{0}\right)=\sum(n / x)\left(x / n_{0}\right)$. We actually obtain the generating function for the moments of $\left(n / n_{0}\right)$. Simple manipulations result in the Cauchy-integral equation $(x) G_{(n / x)}(t ; x)=1 / 2 \pi i \int_{(\Gamma)} G_{(n)}(t / s)$. $E(s ; x) d s / s$ where the $G$ 's represent generating functions for the probability distributions indicated in their subscripts, and $E(s ; x)$ represents an unknown generating function for probabilities $(x / n)$ with respect to $n$. The problem is solved by applying standard generating-function operators to this equation and integrating over the circle $|s|=t$. (Received November 14, 1941.)

89. G. F. McEwen: On the probability that a ratio of random numbers will depart from a harmonic ratio by less than a given amount.

The ratio of two numbers is called harmonic if it equals the ratio of two small integers, one figure numbers, for example. In practical applications both numerator and denominator are subject to error and their ratio accordingly departs from perfect harmony. Accordingly, it is necessary to estimate the probability $P$ of getting by chance a ratio departing less than a given amount from perfect harmony. This problem of determining the proportion, among all possible combinations of numbers, that have a ratio departing from perfect harmony less than this amount, is equivalent to that of finding the probability $P$ that the ratio of any two numbers drawn at random will depart by an amount $X$ or less from the harmonic ratio $N / M$. The solution is $P=2\left(M^{2} / N\right) X /\left[1-(M / N)^{2} X^{2}\right]$, where $N>M$. (Received October 27, 1941.)

\section{TOPOLOGY}

90. W. W. Flexner: Noncommutative chains. II. Preliminary report.

Extending the author's recent work (Duke Mathematical Journal, vol. 8 (1941), pp. 497-505) to a finite convex 3-complex, 2-dimensional noncommutative chains and 
a "homology group" $\Pi_{2}$ are defined. The 2-chains $C$ are products of basic 2-chains $\Omega=a E a^{-1}$ where $E$ is a 2 -cell or its inverse and $a$ is a noncommutative one-chain such that $F \Omega=a(F E) a^{-1}$, where $F E$ is the boundary of $E$, is a continuous closed "path" beginning and ending at a fixed point $\omega$. The group of 2-chains is subject to the relations $C C^{\prime} C^{-1}=(F C) C^{\prime}(F C)^{-1}$. The subgroups $\mathscr{Z}_{2}$, of cycles, and $\mathcal{f}_{2}$, of bounding cycles, are then defined much as in the commutative case. If $\Pi_{2}=Z_{2} / \mathcal{F}_{2}$, it is commutative and subdivision invariant. (Received November 24, 1941.)

\section{D. W. Hall: On a theorem of E. E. Betz.}

In a recent paper (American Journal of Mathematics, vol. 63 (1941), pp. 127-135) E. E. Betz has studied compact locally connected continua $M$ which satisfy the following conditions: (a) no two points of $M$ separate $M$, (b) every simple closed curve $J$ of $M$ separates $M$ into at least two and at most a finite number of complementary domains. He has obtained a rather general accessibility theorem for these continua. Using the result of Betz the present paper shows that if $M$ be any compact locally connected continuum satisfying (a) and (b) and if $G$ is any open subset of $M$, then there is an open subset $R$ of $M$ contained in $G$ which is homeomorphic with an open 2-cell. (Received November 25, 1941.)

\section{R. C. James: Linearly arc-wise connected topological groups.}

The definitions of arc-wise connected, locally arc-wise connected, and simply connected topological spaces are given in terms of a continuous function with real numbers as arguments and values in the topological space. For topological groups, one can add the restriction that the function be linear (additive and continuous) and obtain definitions of linearly arc-wise connected and locally linearly arc-wise connected topological groups. In this paper certain of these properties are used as conditions for a topological abelian group to be a linear topological space. It is shown that a topological abelian group which is simply connected and locally linearly arc-wise connected either has no nonzero elements of finite order or a dense set of elements of the $n$th order (for each $n>1$ ). Several necessary and sufficient conditions for a topological abelian group $T$ to be a linear topological space are found, two of which are: (1) $T$ be connected, locally linearly arc-wise connected, and have no nonzero points of finite order; (2) $T$ be simply connected, locally linearly arc-wise connected, and have a neighborhood of the identity which contains no elements of the second order. (Received October 24, 1941.)

\section{R. C. James: Normable topological abelian groups.}

Several equivalent definitions have been given of bounded sets and convex neighborhoods of a linear topological space. In this paper these concepts are generalized to topological groups, and are used with linear arc-wise connectedness (connectedness by paths defined by a linear function of a real variable) to find conditions that a topological abelian group be normable and that it be a linear topological space. It is shown that a convex, linearly arc-wise connected topological abelian group which has no nonzero elements of finite order is a convex linear topological space, and that a normable, linearly arc-wise connected topological abelian group is a normable linear topological space. A theorem of Komogoroff's on the normability of linear topological spaces is generalized to linearly arc-wise connected topological abelian groups. (Received October $24,1941$. 


\section{Saunders MacLane and Samuel Eilenberg: $A$ theorem on group extensions.}

An abelian group extension of a group $G$ by an abelian group $H$ is customarily represented either by means of a factor set of $H$ in $G$, or by means of a multiplication table for the generators of $H$. The latter depends essentially on the representation of $H$ as a factor group $H=F-B$ of a free group $F$. The exact equivlaence of these two descriptions of a group extension turns out to be important in the study of homology groups (abstract 47-11-487). It may be stated as follows: If $F$ is a free group, and $H=F-B$, then the group Fact $\{G, H\}$ of (abelian) factor sets of $H$ in $G$ modulo the transformation quantities is isomorphic to the group Hom $\{B, G\}$ of all homomorphisms of $B$ into $G$, modulo the subgroup of the homomorphisms of $B$ into $G$ which can be extended to $F$. If $G$ is topological, so is this isomorphism. (Received November 24, 1941.)

\section{Harlan C. Miller: On irreducible continua.}

This paper is concerned with properties of compact continua which are irreducible between some two points, and especially of those which are unicoherent. Some of the results are: If $M$ is a compact atriodic unicoherent continuum, and $H$ and $K$ are proper subcontinua such that $M=H+K$, then $H$ and $K$ are unicoherent. If $M$ is a compact hereditarily decomposable continuum irreducible between some two points, then $M$ contains two mutually exclusive continua, $H$ and $K$, such that (1) in order that $M$ be irreducible between two points it is necessary and sufficient that one of the points belong to $H$ and the other to $K$, and (2) $M-(H+K)$ is strongly connected. If $M$ is a compact hereditarily decomposable continuum irreducible from point $A$ to point $B$, there exists an upper semi-continuous collection, $G$, of mutually exclusive continua filling up $M$ such that if $H$ and $K$ are the elements of $G$ containing $A$ and $B$, respectively, then with respect to its elements, $G$ is an arc from $H$ to $K$; in order that $M$ be unicoherent it is necessary and sufficient that $G$ be a continuous collection. (Received November 25, 1941.)

96. Knox Millsaps: First order differentials of functions with arguments in topological groups and values in topological abelian groups.

A first order differential is defined for a function $F(x)$ with arguments in a topological group $G$ and values in a topological abelian group $A$ and increments in the central subgroup of $G$ with a relativized topology and a generation postulate. The fundamental theorems on uniqueness for increments in the central subgroup, continuity, linear combinations, and the iterative function $K(F(x))$, where $K(y)$, has arguments and values in t.a.g.'s and is $M_{1}$-differentiable (see Michal, Revista de Ciencias), are then proved. (Received October 24, 1941.)

\section{M. E. Shanks: Irreducible transformations of the discontinuum into continua. Preliminary report.}

A continuous transformation $T$ of the Cantor discontinuum $C$ into a continuum is strongly irreducible on a closed subset homeomorphic to $C$. Irreducible transformations of $C$ into locally connected continua are studied in particular with special reference to the one-dimensional case. Certain results are obtained which indicate a simple structure for $T$ in special one-dimensional cases. (Received October 24, 1941.) 


\section{R. H. Sorgenfrey: Some theorems on co-terminal arcs.}

The following results have been established: If $H$ denotes the sum of two arcs with common end points and $K$ denotes their common part, then every component of $H-K$ is a subset of some simple closed curve lying in $\overline{H-K}$. If three arcs have a common end point and no one of them is a subset of any other, then one of them fails to be a subset of the sum of the other two. If three arcs have two end points in common then there are two of them neither of which is a subset of the sum of the remaining two. (Received November 25, 1941.)

99. P. M. Swingle: Topological foundations of an uncertain mathematics.

In this paper a probable origin is given to point sets, as in quantum physics, in a universe of incessantly moving electrons. Philosophical consideration is given to the origin of ideas in such a universe and the conclusion reached that there is little reason for the certainty that exists in traditional mathematics. The nature of a probability topology is considered, a degree of certainty being reached in an abstract topology having an interpretation in what is called a diophantine space, whose points are "cube"-units. (Received October 27, 1941.)

\section{P. A. White: R-regular convergence spaces.}

In this paper a hyperspace $K_{r}$ of a compact metric space $M$ is considered, which consists of all closed locally $\gamma^{s}$-connected $(s \leqq r)$ subsets of $M$. An infinite sequence $\left\{p_{i}\right\} \subset K$ will be said to converge to $p \subset K$ provided the corresponding subsets $\left\{P_{i}\right\}$ of $M$ converge $s$-regularly to $P,(s \leqq r)$. This definition of convergence is used to define limit point, closed set, and open set in the obvious manner. It is shown that the original definition of limit point is equivalent to an open set definition, and that $K$ will be a regular Hausdorff space satisfying the first axiom of countability with these open sets as neighborhoods. It is also shown that unless $M$ is finite, $K$ can be written as the sum of a countable number of sets which are both open and closed, and that not more than one of these sets can be compact. (Received November 24,1941.)

\section{G. W. Whitehead: Homotopy groups of spheres and their rota- tion groups.}

In this paper relations between the homotopy groups of the $n$-sphere $S^{n}$ and those of its rotation group $R_{n}$ are studied. The relationship between these groups is established as follows: to each map $f\left(S^{m} \times S^{n}\right) \subset S^{n}$ there is associated a mapping $f^{*}\left(S^{m+n+1}\right) \subset S^{n+1}$. This operation is a generalization of one introduced by H. Hopf (Fundamenta Mathematicae, vol. 25 (1935), pp. 427-440), and induces a homomorphism of $\pi_{m}\left(R_{n}\right)$ into $\pi_{m+n+1}\left(S^{n+1}\right)$. For $m=1,2$ this homomorphism is an isomorphism. This isomorphism is used to construct a counterexample to a theorem stated without proof by H. Freudenthal (Proceedings, Akademie van Wetenschappen te Amsterdam, vol. 42 (1939), pp. 139-140). In particular, Freudenthal's construction of maps of $S^{2 n-1}$ on $S^{n}$ with Hopf invariant 1 fails if $n \equiv 2(\bmod 4)$. (Received November $24,1941$. 